Authors:

Bradbury, Carl'1. \& Britton, Ingrid². \& Lillie, Kate ${ }^{3}$. \& Wright-White, Helen 4 .

${ }^{1}$ Advanced Practitioner Radiographer (carl.bradbury@uhnm.nhs.uk) (Corresponding author), ${ }^{2}$ Divisional lead GI Consultant Radiologist (ingrid.britton@uhnm.nhs.uk), ${ }^{3}$ Lecturer in Adult Nursing (a.k.lillie@keele.ac.uk). ${ }^{4}$ Academic Development Officer (helen.wright@uhnm.nhs.uk).

1-2Imaging Department - University Hospital of North Midlands, Stoke-on-Trent, United Kingdom ${ }^{3}$ School of Nursing \& Midwifery - Keele University, Stoke-on-Trent, United Kingdom

${ }^{4}$ Research and Development Department - University Hospital of North Midlands, Stoke-on-Trent, United Kingdom.

\title{
Abdominal Radiograph Preliminary Clinical Evaluation Image Test Bank Project.
}




\section{Key words:}

"abdomen”, "projectional radiography", "preliminary clinical evaluation”, "abdominal”, "radiographer", "image bank".

\section{Classifications:}

Radiography, Preliminary Clinical Evaluation, Continuous Professional Development, Audit.

\section{Background}

The focus of radiographer performed Preliminary Clinical Evaluation (PCE) has been on most part, the PCE of musculoskeletal radiographs(1). Abdominal radiographs are often the initial imaging performed on patients attending an emergency department complaining of acute abdominal pain(2). Clinically significant pathologies which may account for abdominal pain that may be visualised on the abdominal radiograph include small bowel obstruction, large bowel obstruction and renal calculi(3). An audit undertaken at the study centre for the period 2013-2017 indicated abdominal radiographs represent on average 3.8\% $(n=2365$ average number of abdominal radiographs per year) of Emergency Department (ED) projectional radiography requests each year. Despite some evidence suggesting that the abdominal radiograph is of limited clinical value $(4,5)$, audit conducted at the study centre demonstrates a sustained number of abdominal radiographs requested by emergency department clinicians between 2013-2017, with no greater than a $\pm 10 \%$ year on year disparity in abdominal radiograph workload. However despite this, only $16 \%$ of imaging departments support radiographer performed abdominal radiograph PCE(1).

Clinicians request abdominal radiographs due to the ease of availability, alongside the simplicity and low cost attributes, as well as the lower ionising radiation exposure when compared to computed tomography scans(6). Further supporting the abdominal radiograph, literature suggests that the abdominal radiograph strengthens the case for further cross-sectional imaging of the abdomen whilst abdominal radiographs may be considered to be an effective triage tool (7-9), referrers need to have an appreciation of the dose implications from additional imaging referrals. The abdominal radiograph is however, often only indicated in specific circumstances due to the low diagnostic yield of the abdominal radiograph when compared with cross-sectional imaging $(3,8,9)$.

This study was based in a large major trauma centre in the Midlands where currently the radiographers participate in a 'red dot' radiographer abnormality detection scheme (RADS). The 'red dot' scheme is the original radiography abnormality signalling system, this system no longer satisfies governance frameworks and is being phased out in favour of PCE schemes(10). The PCE scheme aims to improve the value of the radiographers' contribution to patient management(10). The current RADS at the study centre comprises an agreed scope of practice limited to musculoskeletal radiographs in the ED setting.

The study will establish the sensitivity and specificity with which abdominal radiographs are interpreted by radiographers, and assess whether a significant difference exists in evaluation skill due to experience. It is 
hypothesised that radiographers participating in the study will demonstrate a reasonable sensitivity through selecting an appropriate PCE taxonomy for the abnormal test bank abdominal radiographs. NHS pay scale banding will provide a pseudo measure of experience. Studies suggest that radiographers(11) and medical students(12) have limited confidence in their ability to evaluate the appearance of abdominal radiographs when compared to their perceived ability to interpret musculoskeletal radiographs. Despite this, guidance suggests that a radiographer is well placed to provide a preliminary evaluation of acquired radiographs, to help contribute towards the wider management of the patient $(1,10)$.

Using the Health Research Authority (HRA) decision tool it was concluded that this project did not require submission for HRA or Research Ethical Approval, because the aim was to assess the accuracy with which a current RADS could be extended to include abdominal radiographs. The Research and Development department and the Information Governance team at the study centre approved the commencement of the service evaluation project having been sent a copy of a project implementation outline.

The aim of this study was to determine the accuracy of radiographers in providing PCE taxonomy for abdominal radiographs requested from the ED in order to assess whether it is feasible to extend current practice.

\section{Methods}

A total of 32 diagnostic radiographers, of varied clinical experience, were invited to provide a predetermined PCE taxonomy for an image bank of 30 abdominal radiographs. The abnormal abdominal radiographs included radiographs with evidence of small bowel obstruction, masses, foreign body, toxic megacolon, pneumobilia, hollow viscus perforation, hyperdistention of the urinary bladder, splenomegaly, renal calculus and colitis. No education was provided to the participants prior to taking part in the study. It is, however, acknowledged that the band 7 radiographer group all had prior post graduate level training in the interpretation of abdominal radiographs and/or barium follow through examinations. The PCE taxonomy was based on their perceived clinical urgency of each radiograph. Eleven band 5 radiographers, sixteen band 6 radiographers and five band 7 radiographers took part in the Abdominal Radiograph Image Test Bank Project.

A hypertext mark-up language (HTML) based image test bank was designed with basic image viewing tools including a 'windowing' function and a brief clinical history relating to each radiograph. Each of the 30 abdominal radiographs was displayed on an Eizo RadiForce MX191 picture archiving and communication system (PACS) monitor (size: 19 inches, resolution: 1280 x 1024 pixels) alongside four radio buttons detailing the four possible PCE taxonomies for each abdominal radiograph: urgent clinical attention, requires action, unimportant/incidental finding or no plain film abnormality. Literature suggests that it is reasonable to categorise the findings of abdominal radiographs into these limited number of categories(7). Each reader was allocated 20 minutes to review the 30 radiographs and select an appropriate PCE taxonomy. A timescale was stipulated to better represent the time constraints of PCE in the clinical environment; the timescale allocated is consistent with that of other image interpretation studies (13-15).

Seventeen of the 30 radiographs had some form of abnormality; a high prevalence of abnormal abdominal radiographs was included in the image bank to represent the varied pathologies $(16,17)$ that a radiographer 
may be exposed to in the study centre. The PCE taxonomy selected by the radiographer for each of the 30 abdominal radiographs was compared to the gold standard, which for the purpose of the project is the final radiology report for each of the included abdominal radiographs. The original radiology reports for the positive cases were assigned to one of the four taxonomies, corroborated by two experienced gastrointestinal advanced practitioner radiographers, and this is what participants taxonomies were compared against. To eliminate bias, these gastrointestinal advanced practitioner radiographers did not take part in the image test bank study.

\section{Results}

A total of 32 diagnostic radiographers participated in the Abdominal Radiograph Image Test Bank Project. Eleven band 5 radiographers, 16 band 6 radiographers and 5 band 7 radiographers provided a PCE taxonomy for an image bank of 30 abdominal radiographs. The PCE taxonomy was based on their perceived clinical urgency of each radiograph. Statistical analyses were made possible using Microsoft Excel and International Business Machines (IBM) Statistical Package for the Social Sciences (SPSS) software v25.

A total of 960 opinions were submitted and the number and percent of submitted opinion by gold standard classification are shown in Table 1.

Table1: Submitted Opinion and Gold Standard

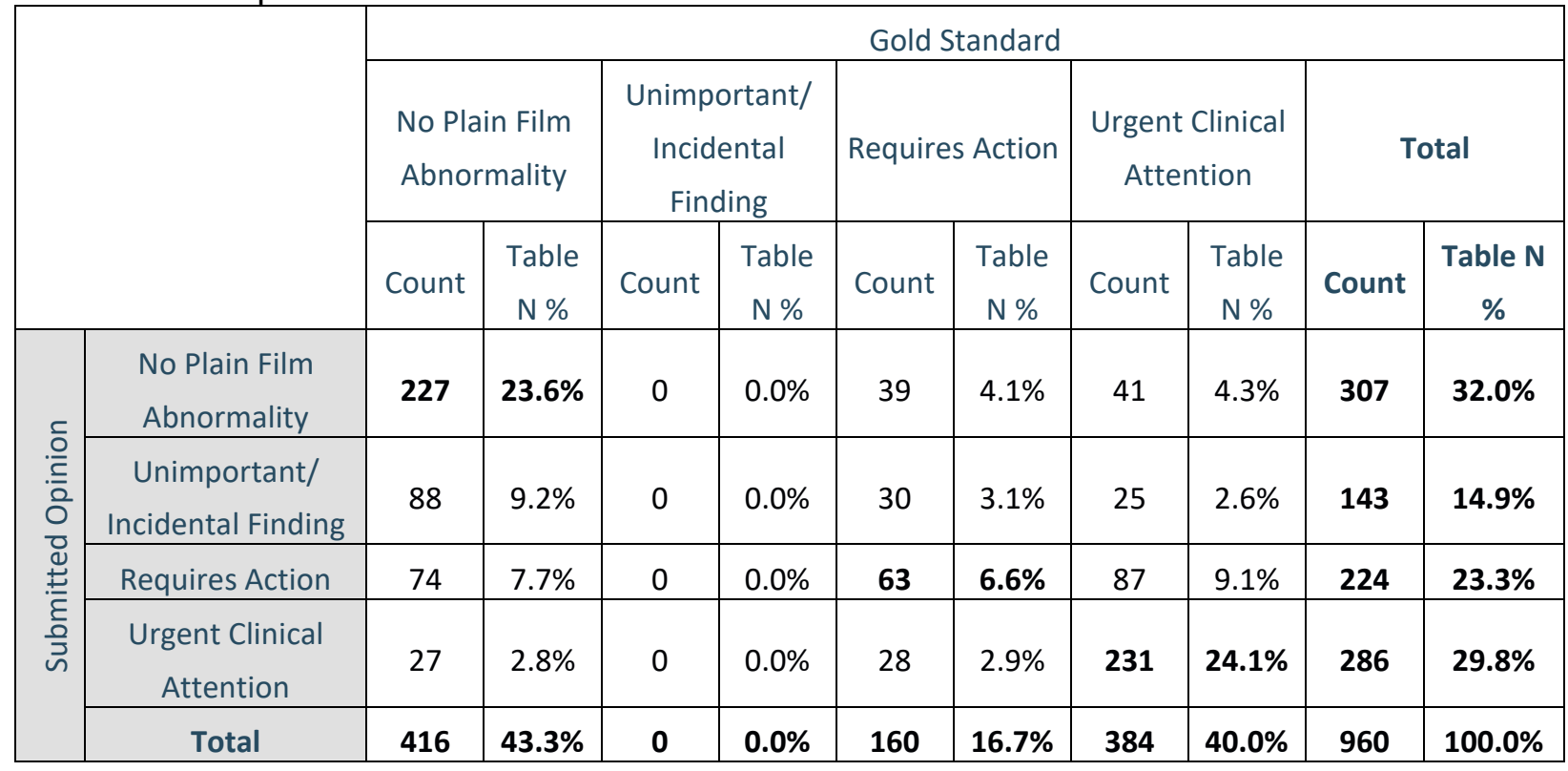

It was noted that no experts classified any of the images as 'unimportant/incidental finding'. Of the 960 radiographs, $544(75.2 \%)$ were categorised as 'abnormal' by the gold standard (either 'requires action' or ‘urgent clinical attention' taxonomy).

In order to calculate sensitivity and specificity, categories were grouped as 'normal' or 'abnormal'. Table 2 shows the contingency table of classification.

Table 2: Opinion versus grouped Gold Standard 


\begin{tabular}{ll|l|r|r|r}
\hline \multirow{2}{*}{$\begin{array}{l}\text { Submitted } \\
\text { Opinion }\end{array}$} & Negative & Count & 315 & 135 & 450 \\
\cline { 2 - 6 } & & $\%$ & $75.7 \%$ & $24.8 \%$ & $46.9 \%$ \\
\cline { 2 - 6 } & Positive & Count & 101 & 409 & 510 \\
\cline { 2 - 6 } & $\%$ & $24.3 \%$ & $75.2 \%$ & $53.1 \%$ \\
\hline Total & Count & 416 & 544 & 960 \\
\hline
\end{tabular}

Overall, radiographers correctly reported an abnormal abdominal radiograph on 409 out of 510 radiographs. Therefore, the estimated sensitivity, or true positive, is $75.2 \%$. Radiographers correctly reported a normal radiograph on 315 out of 450 radiographs, giving an estimated specificity, or true negative, of $75.7 \%$.

The area under a Receiver Operating Characteristic (ROC) curve quantifies the overall ability of the test to discriminate between individuals with an abnormality and those without. Figure 1 shows the ROC curve for all radiographers reporting in this study and the corresponding Area Under the Curve (AUC) shows that radiographers can discriminate between normal and abnormal radiographs $(A \cup C=0.80$, std err $=0.014$, $95 \% \mathrm{Cl} ; 0.772,0.828, \mathrm{p}<0.001)$.

Figure 1: Illustrates the ROC of all radiographers that participated in the study.

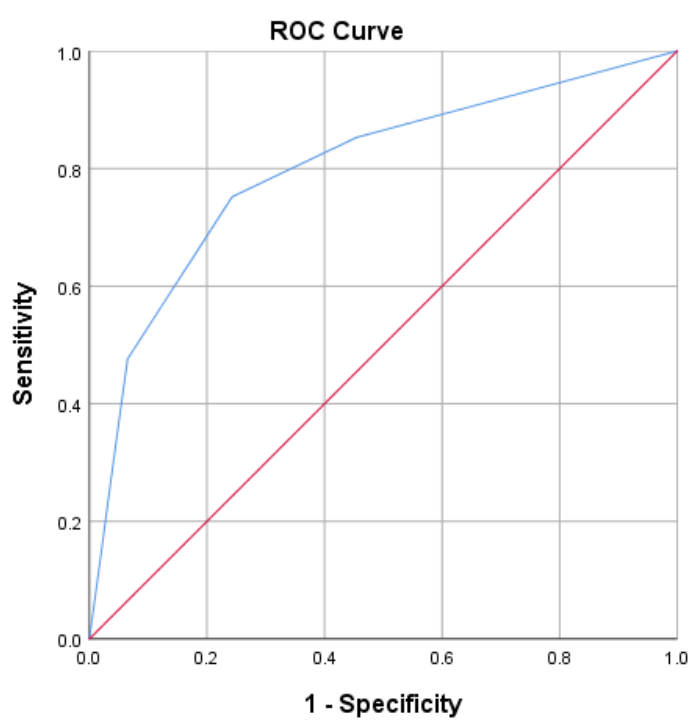

Diagonal segments are produced by ties. 
Table 3 shows the number and percentage of correctly identified abnormal and normal radiographs, the Area Under the Curve, Standard Error, 95\% Confidence Interval and significance value for each group of radiographers split on the basis of NHS pay banding.

\begin{tabular}{|l|c|c|c|c|c|c|c|c|}
\hline & \multicolumn{2}{|c|}{$\begin{array}{c}\text { \#Correct Abnormal } \\
\text { Radiographs (\%) }\end{array}$} & \multicolumn{2}{|c|}{$\begin{array}{c}\text { \#Correct Normal } \\
\text { Radiographs (\%) }\end{array}$} & AUC & Std Error & 95\% Cl & p-value \\
\hline Band 5 & $\frac{136}{187}$ & $72.7 \%$ & $\frac{109}{143}$ & $76.2 \%$ & $77.8 \%$ & 0.026 & $0.727,0.828$ & $<\mathbf{0 . 0 0 1}$ \\
\hline Band 6 & $\frac{209}{272}$ & $76.8 \%$ & $\frac{153}{208}$ & $73.6 \%$ & $80.4 \%$ & 0.02 & $0.764,0.843$ & $<0.001$ \\
\hline Band 7 & $\frac{64}{85}$ & $75.3 \%$ & $\frac{53}{65}$ & $81.5 \%$ & $83.1 \%$ & 0.034 & $0.764,0.899$ & $<0.001$ \\
\hline
\end{tabular}

Table 3 shows that all groups were able to identify between $72 \%$ and $75 \%$ of abnormal radiographs and between $73 \%$ and $81 \%$ of normal radiographs. Almost $25 \%$ of abnormal radiographs were incorrectly identified and almost $20 \%$ of normal radiographs were incorrectly identified. The overall accuracy for all groups ranged from Band 5's AUC $=77.8 \%$ to Band 7's $83.1 \%$, however, a chi-square test of independence was performed to examine the relation between accuracy and experience (Banding). No significant relationship was found between accuracy and banding $\left[X^{2}(2, N=960)=0.831, p=0.660\right]$.

Some noteworthy abdominal radiographs consistently received inaccurate PCE taxonomies by participants; of particular note are the PCE taxonomy selected for radiographs with evidence of colonic mass, pneumobilia and signs of colitis. These radiographs often received an inaccurate PCE taxonomy versus the gold standard.

\section{Discussion}

The results of the study are promising, and demonstrate that on the whole, radiographers are able to distinguish between the abnormal and normal abdominal radiograph through the selection of an appropriate PCE (AUC=80.0\%). The band 7 radiographer group categorised the highest number of accurate PCE statements, however, there was no statistically significant difference in the accuracy of PCE selection between the radiographers of different pay bands included in the study. Despite these promising results, almost a quarter of radiographs were still incorrectly classified, suggesting the need for further abdominal radiograph image interpretation education.

As a condition of health and care professions council (HCPC) registration all radiographers must demonstrate the ability to understand common pathologies, and to differentiate between the normal and abnormal appearances present on radiographs(18). Furthermore the Society and College of Radiographers (SCoR) explain that preliminary clinical evaluations should form part of the core professional scope of practice expected of a radiographer(10). The results of this study support the ability of radiographers to use their knowledge and skill to accurately discriminate between abdominal radiographs with pathology and those without. 
There are acknowledged limitations associated with the methodology, these include a degree of fallibility related to the allocation of a 'gold standard' PCE statement relative to the 'gold standard' abdomen radiograph report, and this fallibility is influenced by the advanced practitioner radiographers' perception of the clinical urgency. The radiographer applying the PCE statement is limited by their inability to view clinical notes and complimentary imaging that may have been available at the time the abdominal radiograph was acquired; consideration of the inclusion of this facility could be made for the future development of image test banks. A comprehensive clinical history is often not included for emergency department referrals(19); this may inhibit the ability of a radiographer to accurately provide a PCE for a radiograph.

A high prevalence of abnormal abdominal radiographs was included in the image test bank, representing $57 \%$ of the radiographs included. Limited literature related to abdominal radiograph abnormality yield in the ED suggest approximately $12 \%$ of radiographs have abnormal or non-specific findings(3,20). A high proportion of abnormal radiographs was included in the image test bank to maximise exposure of the participants to a variety of abnormal radiographs $(16,21)$ relating to abdominal pathologies that may be encountered in the emergency department of the study centre. However, previous studies have demonstrated that high prevalence image test banks tend to improve the readers sensitivity $(16,17,22)$, which is borne in mind when interpreting the results of this study. The mean sensitivity and specificity values achieved by participants in this study is comparable to the values achieved by participants in musculoskeletal PCE image test bank projects $(13,21)$.

It would be prudent to further research the effectiveness of abdominal radiograph PCE with a greater number of participants using an image test bank with a lower prevalence of positive radiographs. This will better represent the positive yield of abdominal radiograph pathology that one would expect to encounter in the clinical environment. Furthermore, this will reduce chance of the phenomena of prevalence bias where a high sensitivity is achieved by the participant in a high positive pathology prevalence environment(16). This would then allow for the calculation of positive predictive and negative predictive values (PPV/NPV) which are dependent on prevalence rates in the population.

A future study may benefit from a pre and post additional abdominal radiograph interpretation education arm to the study. Studies focussing on musculoskeletal PCE have demonstrated the value of the integration of education with image interpretation schemes(23-26). Each study shared a common theme that education can positively influence the image interpretation skill of the participant.

Prior to exploring an expansion of practice, further consideration should be made relating to the introduction of targeted education for a number of abdominal radiographs that consistently received inaccurate PCE taxonomies. These included radiographs with evidence of colonic mass, pneumobilia and signs of colitis. Studies suggest that inflammatory conditions can be overlooked on abdominal radiographs due to poor soft tissue contrast resolution(4), this may account for the inaccuracy of PCE taxonomies received. Pneumobilia is better assessed on cross-sectional imaging(27); the aetiology of this finding does not always indicate the 
presence of pathology; thus the finding may well be incidental, hence this may have created PCE taxonomy selection ambiguity.

\section{Conclusion}

This study suggests that radiographers are able to accurately distinguish between normal and abnormal abdominal radiographs. Furthermore, there was no evidence to suggest that there exists a significant difference in ability to interpret radiographs, based on experience. However, when assessing less obvious appearances such as colonic mass and signs of colitis, sensitivity is reduced illustrating an area for additional training. This study provides evidence to support an expansion of current practice in the implementation of a scheme of abdominal radiograph PCE. Further research would be beneficial, with a larger cohort of radiographers, and an integration of education to demonstrate the value of continuous professional development in relation to improving a radiographer's ability to apply interpretative skill to the selection of an appropriate abdominal radiograph PCE taxonomy. 
1. The Society and College of Radiographers. Diagnostic Radiography: A Survey of the Scope of Radiographic Practice 2015 [Internet]. 2015. p. 1-48. Available from:

https://www.sor.org/sites/default/files/document-versions/2017-05-

17_diagnostic_scope_of_practice_2015_-_final.pdf

2. Loo JT, Duddalwar V, Chen FK, Tejura T, Lekht I, Gulati M. Abdominal radiograph pearls and pitfalls for the emergency department radiologist: a pictorial review. Abdom Radiol. 2017;42(4):987-1019.

3. Jackson K, Taylor D, Judkins S. Emergency department abdominal x-rays have a poor diagnostic yield and their usefulness is questionable. Emerg Med J. 2011;28(9):745-9.

4. Alshamari M, Norrman E, Geijer M, Jansson K, Geijer H. Diagnostic accuracy of low-dose CT compared with abdominal radiography in non-traumatic acute abdominal pain: prospective study and systematic review. Eur Radiol. 2016;26(6):1766-74.

5. Gans SL, Pols MA, Stoker J, Boermeester MA. Guideline for the diagnostic pathway in patients with acute abdominal pain. Dig Surg. 2015;32(1):23-31.

6. Gans SL, Stoker J, Boermeester MA. Plain abdominal radiography in acute abdominal pain; past, present, and future. Int J Gen Med. 2012;5(1):525-33.

7. Mohamed H, Ismail B, Malik A. Will Plain Abdominal Radiographs become Obsolete ? Radiol Open. 2017;2(2):32-7.

8. The Royal College of Radiologists. iRefer Guidelines: Making the best use of clinical radiology [Internet]. iRefer Guidelines: Making the best use of clinical radiology. 2017 [cited 2018 Aug 24]. Available from: https://www.irefer.org.uk/

9. Artigas Martín JM, Martí De Gracia M, Rodríguez Torres C, Marquina Martínez D, Parrilla Herranz P. Radiografía del abdomen en Urgencias. Una exploración para el recuerdo? Radiologia [Internet]. 2015;57(5):380-90. Available from: http://dx.doi.org/10.1016/j.rx.2015.06.002

10. SCoR. Preliminary Clinical Evaluation and Clinical Reporting by Radiographers: Policy and Practice Guidance [Internet]. London: SCoR; 2013. p. 1-11. Available from:

https://www.sor.org/printpdf/book/export/html/9197

11. Lancaster A, Hardy M. An investigation into the opportunities and barriers to participation in a radiographer comment scheme, in a multi-centre NHS trust. Radiography [Internet]. 2012;18(2):1058. Available from: http://dx.doi.org/10.1016/j.radi.2011.08.003

12. Jacob J, Paul L, Hedges W, Hutchison P, Cameron E, Matthews D, et al. Undergraduate radiology teaching in a UK medical school: A systematic evaluation of current practice. Clin Radiol [Internet]. 2016;71(5):476-83. Available from: http://dx.doi.org/10.1016/j.crad.2015.11.021

13. Wright C, Reeves P. RadBench: Benchmarking image interpretation skills. Radiography [Internet]. 2016;22(2):e131-6. Available from: http://dx.doi.org/10.1016/j.radi.2015.12.010 
14. McLaughlin L, McConnell J, McFadden S, Bond R, Hughes C. Methods employed for chest radiograph interpretation education for radiographers: A systematic review of the literature. Radiography [Internet]. 2017;23(4):350-7. Available from: https://doi.org/10.1016/j.radi.2017.07.013

15. Semakula-Katende, N. S., Andronikou, S., \& Lucas S. Digital platform for improving non-radiologists' and radiologists' interpretation of chest radiographs for suspected tuberculosis-a method for supporting task-shifting in developing countries. Pediatr Radiol [Internet]. 2016;46(10):1384-91. Available from: http://dx.doi.org/10.1007/s00247-016-3630-y

16. Pusic M V., Andrews JS, Kessler DO, Teng DC, Pecaric MR, Ruzal-Shapiro C, et al. Prevalence of abnormal cases in an image bank affects the learning of radiograph interpretation. Med Educ. 2012;46(3):289-98.

17. Nocum DJ, Brennan PC, Huang RT, Reed WM. The effect of abnormality-prevalence expectation on naïve observer performance and visual search. Radiography [Internet]. 2013;19(3):196-9. Available from: http://dx.doi.org/10.1016/j.radi.2013.04.004

18. HCPC. Standards of proficiency - Radiographers [Internet]. London: HCPC; 2013. p. 1-20. Available from: http://www.hpcuk.org/assets/documents/10000DBDStandards_of_Proficiency_Radiographers.pdf

19. Davies S, George A, Macallister A, Barton H, Youssef A, Boyle L, et al. "It's all in the history": A service evaluation of the quality of radiological requests in acute imaging. Radiography [Internet]. 2018;24(3):252-6. Available from: https://doi.org/10.1016/j.radi.2018.03.005

20. Zeina AR, Shapira-Rootman M, Mahamid A, Ashkar J, Abu-Mouch S, Nachtigal A. Role of plain abdominal radiographs in the evaluation of patients with non-traumatic abdominal pain. Isr Med Assoc J. 2015;17(11):678-81.

21. Hardy M, Flintham K, Snaith B, Lewis EF. The impact of image test bank construction on radiographic interpretation outcomes: A comparison study. Radiography [Internet]. 2016;22(2):16670. Available from: http://dx.doi.org/10.1016/j.radi.2015.10.010

22. Wolfe JM, Horowitz TS, Van Wert MJ, Kenner NM, Place SS, Kibbi N. Low Target Prevalence Is a Stubborn Source of Errors in Visual Search Tasks. J Exp Psychol Gen. 2007;136(4):623-38.

23. Stevens BJ, Thompson JD. The impact of focused training on abnormality detection and provision of accurate preliminary clinical evaluation in newly qualified radiographers. Radiography. 2018;24(1):47-51.

24. Wright C, Reeves P. Image interpretation performance: A longitudinal study from novice to professional. Radiography [Internet]. 2017;23(1):e1-7. Available from: http://dx.doi.org/10.1016/j.radi.2016.08.006

25. Stevens BJ, White N. Newly qualified radiographers' perceptions of their abnormality detection abilities and the associated training they received at undergraduate level. Radiography. 
2018;24(3):219-23.

26. Hargreaves J, Mackay S. The accuracy of the red dot system: can it improve with training? Radiography. 2003;9(4):283-9.

27. Baron TH, Kozarek RA, Carr-Locke DL. ERCP. USA: Elsevier Health Sciences; 2013. 